\title{
On the Specification of EtE QoS Control
}

\author{
J.B. de Meerl \\ GMD-FOKUS Berlin D-10623, Hardenbergplatz 2, Germany \\ email: jdm@fokus.gmd.de
}

\begin{abstract}
:
Continuous media differ in their nature from discrete media, in that streams provide with a flat structure of data or even with loss of structure instead of single highly structured multityped discrete data units. Streams use simple communication concepts, since they are directed flows. Discrete media however, obey in most cases a complicated communication protocol. Streams are mass transportation vehicles, whereas discrete media cope with less mass of data but usually with more information about a state change of the communicating components. End-to-end control can be achieved by sensoring the effect of system activities and by feeding them back to the related input. Controlling signals will be issued when certain invariants of the system get violated. Models of which obey with components of a continuously operating system, a continuously sensoring and discretely decision-taking component. Whereas the continuous system is forward-oriented, the sensoring and decision-taking components are backwards. A synthesis of these two aspects provides with the basic end-to-end QoS control architecture.
\end{abstract}

\section{Keywords:}

End-to-End QoS Control, Control Systems, Stream Engineering, Monitoring

\section{INTRODUCTION}

One of the most important concept in system engineering is to permanently observe and control an open behaviour to achieve best qualities of services. Here behaviour is called open because the system's background activities will have impacts to the service provision. The 'background noise' may cause delays, bandwidth crashes, or unacceptable jitter during transmission etc. Hence, a major desire in continuously operating systems is the permanent control of the quality of operation and optimal use of resources. One of the first experiments with flow engineering mechanisms have been resulted from the realisation of the "multimedia enhanced transport system" (Campbell etal 1996). The system comprises engineering components to schedule flows, to shape flows according to the token bucket scheme, to filter jitter and to control quality adaptations. A more specification-oriented approach of resource adaptations to QoS constraints has been presented in (Franken etal, 1996).

\section{LIQUid-LEVEL MONITORING PARADIGM}

The paradigm of the "water-level monitor" from (Alur etal, 1996) is very suitable for introducing and reasoning about continuous end-to-end QoS control because the paradigm comprises with the appropriate componental architecture. The liquid-level monitor system consists of a liquid container with a constant sink and a controlled source. The effect of the continuously operating sink and source can be monitored at the liquid level. Now, the liquid level $Y(t)$ must be controlled by switching the source on and off, such that the liquid level is in between of some upper $U B$ and lower bound $L B$. Furthermore, switching on and off the

1. This work is partly supported by the European project AC237. 
source effects the volume of the liquid container after a delay of e.g. 1 second. This paradigm is drafted in figure (1) and consists of the 4 components: source, container, monitor and sink. The source is characterized by the stream $Q_{i}(t)$ conveyed by a server, e.g. a pump and a delay when the effect of the server gets observable; the container is characterized by the conveyed volume, composed from the current liquid level $Y(t)$ and the container's size $A$; the monitor is characterized by the system invariants and thresholds not to be violated resp. to generate control signals to the source; and finally the sink is characterized by his characteristic size $a_{o}$ which allows the stream $Q_{o}(t)$ to flow out of the container constantly.

\section{STREAM BALANCING APPROACH}

By balancing the input and output streams of the container according to (Schlitt 1993, JdeMeer 1996) we get the accumulated volume by equation $V \dot{(t)}=Q_{i}(t)-Q_{o}(t)(1)$. Independent from the source behaviour the output stream is assumed to be constant.

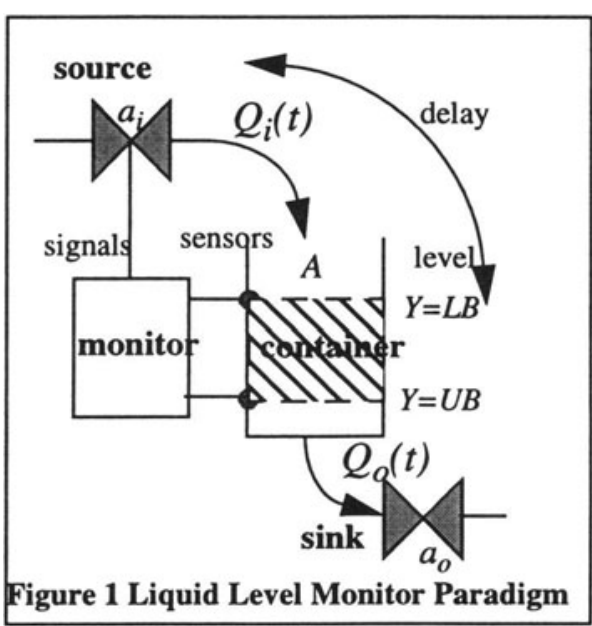

The Liquid-level Monitoring System can easily be translated towards a multi-media servicing system. The components 'source', 'sink' and 'container' are the multi-media producing and consuming components with accumulating facilities in between. The multi-media producing and consuming components usually operate independently, i.e. generating the streams $Q_{i}(t)$ and $Q_{o}(t)$ respectively. Hence, the network must be able to provide with storing facilities characterized by the size $A$ of units to be stored, e.g. pages or bytes, but likely different from the units $a_{o}$ consumed by the sink.

From equation (1) the stream-balancing equation and by replacing the network's output stream $Q_{o}(t)$ by the assumed constant speed of the sink stream $v_{o}$ the differential equation describing the actual accumulation level $Y(t)$ of the network storing capacity is as given in equation (2): $Y \dot{(t)}=-\frac{a_{o}}{A} \cdot v_{o}+\frac{1}{A} \cdot Q_{i}(t)(2)$

Now, the monitor has to control the level of stored units in the network, such that some given upper $U B$ and lower bound $L B$ are never exceeded. In order to deal with the delay requires by the monitor an upper $U T h$ and a lower threshold $L T h$ that mark the filling respectively emptying capacities during delay. Since, in the paradigm the delay shall be a second and the source speed is assumed to be 30 frames per second and the sink shall be 20 frames per second, the upper and lower thresholds and bounds derive to: $U B=100, U T h=90$, $L T h=40, L B=20$ frames. The monitor comprises with the functionality of controlling the system invariants by permanently sensoring characteristic variables, e.g. $Y(t)$, and by issuing of control signals, e.g. "server-on" or "server-off" when certain thresholds have been reached. Similar, the network component is modelled by his capability to accumulate input and output streams. The sink component has a constant stream behaviour, characterized by the through- 
put of the display of presenting units of size $a_{o}$ with the speed of $v_{o}$.

In the paradigm, the output stream $Q_{o}$ is said to be constant, the input stream is not. Now, by integrating equation (2) and by replacing the input stream $Q_{i}(t)$ by $a_{i} / t$ with the operation time $t$ per frame $a_{i}$, we get able to calculate the accumulation level $Y$ of the network buffer for a given time period of $t$ seconds.

$$
Y=\int_{1}^{t+1}\left(-Q_{o}+\frac{a_{i}}{t}\right) d t=-Q_{o} t+\left.a_{i} \cdot \ln t\right|_{1} ^{t+1}=-Q_{o} t+a_{i} \cdot \ln (t+1)
$$

. Notice, in order to simplify the solution of the determinate integral the lower bound of the time interval has been chosen to be 1 . The solution describes the streaming behaviour for a time interval of e.g. $l$ to $t+l$ seconds with frame generation at the server site as a function over time. For example, the monitor could signal to increase or lower the frame generation speed.

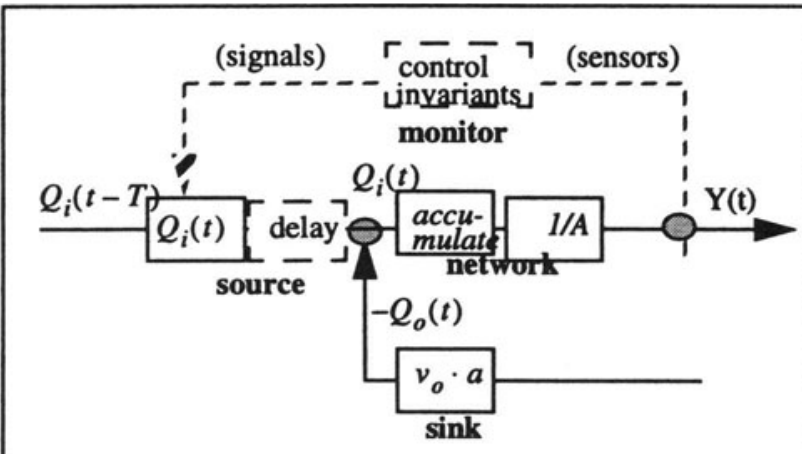

Figure 2 Continuous Stream Model
From equation (2) we are able to immediately derive the componental architecture of the monitor paradigm as given in figure 2. The components of the system, i.e. source, network and sink obey stream-manipulating functionalities of the equation (2). The integral operator defines the accumulation, the stream balancing the subtraction point, the openness of a stream the independent

resp. constant behaviour and $Y(t)$ the measurement (sensoring) point.

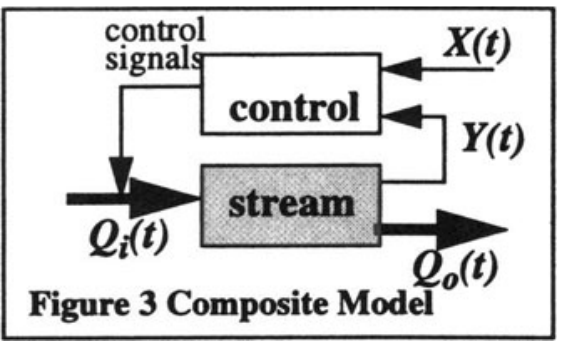

able $Y(t)$ is obtained and will be fed back into the control path. The control component monitors the continuous level function $Y(t)$ and time function $X(t)$ and produces appropriate control signals in order to steer the server. The variables $X(t)$ and $Y(t)$ are 'valuated' by the control component. Whereas $Y(t)$ represents the level of unit accumulation in the system 
buffer $X(t)$ represents the explicit and continuous evolution of time in the control model. An example of decision-taking activity is: 'if $y=U T h$ then signal(server-on)'. Examples of continuous activities are the system's accumulation between the lower bound $L B$ and the upper threshold UTh: 'while $y<U T h$ then incr $(y+10)$ ' or between the upper bound $U B$ and lower threshold LTh: 'while $y>$ LTh do incr $(y-20)$ ' or the system's delay: 'while $x<$ delay do $\operatorname{incr}(x+1)$, incr $(y+10)$ '.

\section{CONCLUSIONS}

A combination of the continuous stream model with the control model to a composite model consisting of the streaming path of $Q_{i}(t) \rightarrow\left(Q_{o}(t), Y(t)\right)$ and of the feed-back control path $((Y(t), X(t)) \rightarrow($ signals $))$ gains the appropriate ent-to-end QoS architecture. It seems to be very adequate because the composite model allows the synthesis of both the capturing of continuously changing system activities with discrete decision taking of the control part. The discrete approach of the control model is suitable to represent the decision-taking machine. In contrast, streams obey duration, capacity, and direction and thus provide with quantitative properties that vary over time. Both systems the discrete one and the continuous one can be connected without further translations. And infact, the view of the system shall be taken separately from the view onto it. At the final end the viewpoint approach of ODP (IS 10746, 1995) gets convincingly achieved. However, it must be critically remarked that the presented approach has not yet been evaluated against a sophisticated multi-media service. But, as shown, control theory fits nicely with the requirements of end-to-end QoS Control and gives powerful means on hand to reason about designed QoS Architectures.

\section{REFERENCES}

A.Campbell, G.Coulson (1996) A QoS Adaptive Multimedia Transport System: Design, Implementation and Experience, DSE Journal.

L.J.N.Franken, B.R.H.M.Haverkort (1996) Quality of Service Management and Monitoring Techniques, DSE Journal

Jan de Meer (1996) Formal Handling of Streams in MM Environments. Proceedings of the workshop of Application of Formal Methods to System Development, Montreal, 2.-4. October 1996, pp.191-200.

ISO/IEC IS/DIS 10746 IT - Basic Reference Model of Open Distributed Processing Part 1 4.

H.Schlitt (1993) Regelungstechnik - Physikalisch orientierte Darstellung fachübergreifender Prinzipien, Vogel-Verlag, ISBN 3-8023-1483-2

R.Alur, C.Courcoubetis, N.Halbwachs, T.A.Henzinger, P-H.Ho, X.Nicollin, A.Olivero, J.Sifakis, S.Yovine (1995) The Algorithmic Analysis of Hybrid Systems, Theoretical Computer Science 138, pp. 3-34 